Complex potential and its effects on bound states

This article has been downloaded from IOPscience. Please scroll down to see the full text article. 1995 Phys. Scr. 51146

(http://iopscience.iop.org/1402-4896/51/2/004)

View the table of contents for this issue, or go to the journal homepage for more

Download details:

IP Address: 139.165.107.21

The article was downloaded on 05/12/2011 at 16:11

Please note that terms and conditions apply. 


\title{
Complex Potential and its Effects on Bound States
}

\author{
J. Cugnon and P.-B. Gossiaux \\ Université de Liège, Institut de Physique au Sart Tilman, Bâtiment B.5, B-4000 LIEGE 1, Belgium
}

Rereived January 10, 1994 ; in revised form June 20, 1994 ; accepted August 25, 1994

\begin{abstract}
The coupling of bound states in a single-particle effective Hamiltonian through the addition of a negative definite imaginary potential is studied, both in a stationary and in a time-dependent formalism. Corresponding physical cases where this coupling is relevant are exhibited. Properties of the imaginary coupling matrix in the representation of the eigenstates of the real Hamiltonian are investigated. Consequences of these properties on the level widths and level shifts are exhibited on the two level case. Non trivial results, like forbidden values of the widths and level attraction are underlined. The conditions for the validity of the weak coupling approximation are examined. In the time-dependent problem, the typical time evolution patterns are illustrated both in the two level case and in the more realistic case of charmonium decay. When the initial state is an eigenstate of the real part of the Hamiltonian, it is shown that mixing of decay modes and quantum interference arise as consequences of the non diagonal imaginary coupling. Finally, the non locality of the imaginary potential corresponding to a diagonal imaginary coupling matrix in the state representation is also briefly studied and illustrated
\end{abstract}

\section{Introduction}

It is very common in the non relativistic quantum mechanical description of two-body scattering or of the widths of (quasi) bounds states, to account for the loss of flux or of probability, due to the neglect of an explicit treatment of some channels, by means of an optical model potential with an imaginary part. A very popular example is provided by the description of the elastic scattering of hadrons by nuclei, using an optical model with a complex potential, the imaginary part of which coming from the loss of flux due to the coupling to inelastic channels [1]. It is customary to analyse the data with a local imaginary potential, in spite of the fact that it can be shown on very theoretical grounds $[2,3]$ that the optical model potential is (weakly) non local and energy dependent. We will consider this optical model, namely the addition of an imaginary part to an effective one-body Hamiltonian, for bound states. We will be a little bit more general than usual, requiring the imaginary potential to be negative definite, allowing it to be local or non local. This model is usually studied, for stationary problems, by solving the static Schrödinger equation in $r$-space. When dealing with time-dependent problems, one would rather be tempted to work in the basis of the eigenstates of the real part of the Hamiltonian, at least when these eigenstates are easily constructed (like in the Coulomb case). Indeed, the problem then arises to solve a set of first order differential equations (instead of solving the time-dependent Schrödinger equation in $r$-space, which is a parabolic partial differential equation). What we want to do here is to exhibit the relationship between the two descriptions (in $r$-space and in the state representation) and, in particular, to study the properties of the coupling imaginary matrix (in the state representation) corresponding to a negative definite imaginary potential. We want also to investigate briefly the consequences of these properties on those of the bound states, both in a stationary and a time-dependent picture.

\section{Schrödinger equation in configuration space and in state representation}

To be specific, we start with the time-dependent Schrödinger equation

$$
\begin{aligned}
i \hbar \frac{\partial \psi(\boldsymbol{r}, t)}{\partial t}= & \left(-\frac{\hbar^{2}}{2 m} \Delta+V(\boldsymbol{r})\right) \psi(\boldsymbol{r}, t)-\mathrm{i} \\
& \times \int \mathrm{d}^{3} r^{\prime} W\left(\boldsymbol{r}, \boldsymbol{r}^{\prime}\right) \psi\left(\boldsymbol{r}^{\prime}, t\right),
\end{aligned}
$$

where $W\left(\boldsymbol{r}, \boldsymbol{r}^{\prime}\right)$ is positive definite. We here restrict the discussion to (quasi) bound states. This framework applies to many problems in hadron physics as well as in atomic physics. For instance, eq. (2.1) is appropriate to describe the evolution of a $\pi^{-}$meson [4] or a $\mathrm{K}^{-}$meson [5] bound to a nucleus in a Coulomb orbit, $W$ (in general local in these cases) accounting then for the possible absorption of the meson due to strong interactions. A similar description is applicable to an antiproton bound by Coulomb forces to a nucleus, and to the protonium system [6]. Still another case corresponds to the charmonium system where $V(\boldsymbol{r})$ is the quark-antiquark potential and a local $W$ stands for the coupling to the $\mathrm{D}-\overline{\mathrm{D}}$ channels $[7,8]$. In atomic physics, some aspects of the evolution of autoionization states [9] can be handled by eq. (2.1).

Instead of solving eq. (2.1) in $r$-space, one may be tempted to use a representation based on the stationary eigenstates of the real part, $H_{0}$, of the Hamiltonian contained in eq. (2.1):

$H_{0} \psi_{k}=\left(-\frac{\hbar^{2}}{2 m} \Delta(r)\right) \psi_{k}=E_{k} \psi_{k}$

Assuming that the stationary eigenstates form a complete set, eq. (2.1) is then equivalent to the following set of equations

$\dot{c}_{k}=-\frac{\mathrm{i}}{h} E_{k} c_{k}-\frac{1}{\hbar} \sum_{j} W_{k j} c_{j}$,

where

$W_{k j}=W_{j k}=\left\langle\psi_{k}|W| \psi_{j}\right\rangle$. 
Searching for stationary states of Hamiltonian (2.1) would, in this representation, amount to solve the eigenvalue problem

$\left(E_{k}-E\right) c_{k}-\mathrm{i} \sum_{j} W_{k j} c_{j}=0$.

We want to address to ourselves the following questions: (a) what are the general properties of the quantities $W_{k j}$, corresponding to a negative definite, imaginary potential in $r$-space?; (b) what are the conditions for using the so-called weak coupling formula, corresponding to the neglect of the off-diagonal elements of $W_{k j}$ ?; (c) can one derive analytical formula from the strong coupling case, at least in simple situations?; (d) what is the effect of the matrix $W_{k j}$ on the characteristic time evolution of the coefficients $c_{k}(t)$, defined in eq. (2.3)?

To make our motivation clearer, we do not pretend here to analyze all the physical effects linked with the imaginary part, as the underlying physical problem amounts to diagonalize a Hamiltonian with a real coupling in a larger space, but we rather want to investigate primarily the mathematical properties of the eigenvalue problem (2.5), which, as we said, is often encountered in physics, because the many channel problem is often intractable. In particular, we want to point out the conditions that the quantities $W_{k j}$ should satisfy and that one should enforce if one intended to modelize the coupling by eq. (2.5) from the very beginning, requiring however that this corresponds to the physical situation of a local negative definite imaginary potential. Nevertheless, we will also point out some physical consequences of the imaginary coupling.

We will also pay some attention to the somehow reverse problem. Assume that in the basis of the functions $\psi_{k}$ defined in (2.2), the absorption is diagonal, i.e. that one has the following set of equations, for the stationary problem (one may easily generalize to the time-dependent problem).

$\left(E_{k}-E\right) c_{k}-i \frac{\Gamma_{k}}{2} c_{k}=0$,

where the $E_{k}$ 's are real. Translated into $r$-space, this equation writes

$\left[-\frac{h^{2}}{2 m} \Delta+V(\boldsymbol{r})\right] \psi(\boldsymbol{r})-\mathrm{i} \int W\left(\boldsymbol{r}, \boldsymbol{r}^{\prime}\right) \psi\left(\boldsymbol{r}^{\prime}\right) \mathrm{d}^{3} r^{\prime}=E \psi(\boldsymbol{r})$,

with

$W\left(r, r^{\prime}\right)=\sum_{j} \frac{\Gamma_{j}}{2} \psi_{j}(\boldsymbol{r}) \psi_{j}\left(\boldsymbol{r}^{\prime}\right)$

In other words, if the "absorption is diagonal" in the state representation, it is in general non local in $r$-space. We will investigate the following questions: (a) what are the general properties of the quantity $W\left(\boldsymbol{r}, \boldsymbol{r}^{\prime}\right)$ defined by eq. (2.8)?; (b) what would be the conditions for this quantity to be local or almost local?

\section{Properties of the quantities $W_{k j}$}

If the potential $W\left(\boldsymbol{r}, \boldsymbol{r}^{\prime}\right)$ is given, the quantities $W_{k j}$ can be calculated easily. However, it is worthwhile to find out the properties of the matrix $W_{k j}$. This may be helpful in cases where one would like to use the optical model in the formulation (2.5) from the beginning and where the imaginary part is not known in full detail and thus has to be postulated.

We consider (semi) positive definite $W\left(\boldsymbol{r}, \boldsymbol{r}^{\prime}\right)^{1}$, i.e. functions which satisfy

$\int d^{3} r \int d^{3} r^{\prime} \psi^{*}(r) \boldsymbol{W}\left(\boldsymbol{r}, \boldsymbol{r}^{\prime}\right) \psi\left(\boldsymbol{r}^{\prime}\right) \geqslant 0$,

for any $\psi(r)$, because it leads to a decrease of the norm of the wave function $\psi(\boldsymbol{r}, t)$ in eq. (2.1), or to complex eigenvalues of the energy with negative imaginary parts in the stationary Schrödinger equation. For a positive definite operator, the matrix representing this operator in any basis is also positive definite [10]. Therefore, all the principal minors are positive definite [11]:

$W_{j j} \geqslant 0$,

$W_{j j} W_{k k}-W_{k j}^{2} \geqslant 0$

$W_{j j} W_{k k} W_{l l}+W_{j k} W_{k l} W_{l j}+W_{j l} W_{k j} W_{l k}$

$$
-W_{l j} W_{k k} W_{j l}-W_{k i} W_{l k} W_{j j}-W_{l l} W_{j k} W_{k j} \geqslant 0,
$$

$\cdots$

Let us remark that the sufficient condition for $W_{k j}$ to be positive definite is that the leading principal minor (i.e. the one in the upper left corner) of any rank is positive.

If $W$ is positive definite and local, i.e. if $W\left(r, r^{\prime}\right)=W(r) \delta(r$, $r^{\prime}$ ), condition (3.3) can also be obtained from Schwarz's inequality for integrals with a weighted norm [12]. Indeed, any matrix element $W_{k j}$ is a scalar product of $\psi_{k}$ by $\psi_{j}$ with the positive weight $W(r)$. In this case, additional relations may be derived. One has

$\sum_{k} W_{k k}=\infty$,

which proceeds from the invariance of the trace (let us recall that a local operator in configuration space has an infinite trace). One also has

$W_{k k} \leqslant \max W(\boldsymbol{r})$,

as the wave functions $\psi_{k}$ can be taken real, and

$\sum_{j} W_{k j}^{2}=\int \psi_{k}(r) W^{2}(\boldsymbol{r}) \psi_{k}(\boldsymbol{r}) \mathrm{d}^{3} r$,
$\sum_{j} W_{k j}^{2} \leqslant \max _{\boldsymbol{r}} W^{2}(\boldsymbol{r})$,

$\sum_{j}\left(E_{j}-E_{k}\right) W_{k j}^{2}=\frac{\hbar^{2}}{2 m} \int \psi_{k}(\nabla W)^{2} \psi_{k} \mathrm{~d}^{3} r$

$\sum_{j}\left(E_{j}-E_{k}\right) W_{k j}^{2} \leqslant \frac{\hbar^{2}}{2 m} \max _{r}(\nabla W)^{2}$.

Relation (3.8a) can be obtained by the double commutator technique [13] or progenitor technique [14], which generate energy weighted sum rules, of which (3.8a) is just an example. In that particular case, it is easy to show that the 1.h.s. of (3.8a) is simply

$\sum_{j}\left(E_{j}-E_{k}\right) W_{k j}^{2}=\frac{1}{2}\left\langle\psi_{k}\left|\left[W,\left[H_{0}, W\right]\right]\right| \psi_{k}\right\rangle$.

1 The expressions below are written for a semi-positive definite operator $W$, although we will not repeat the prefix "semi". Of course, for a truly positive operator the symbol "larger or equal" should be replaced by the symbol "larger". 


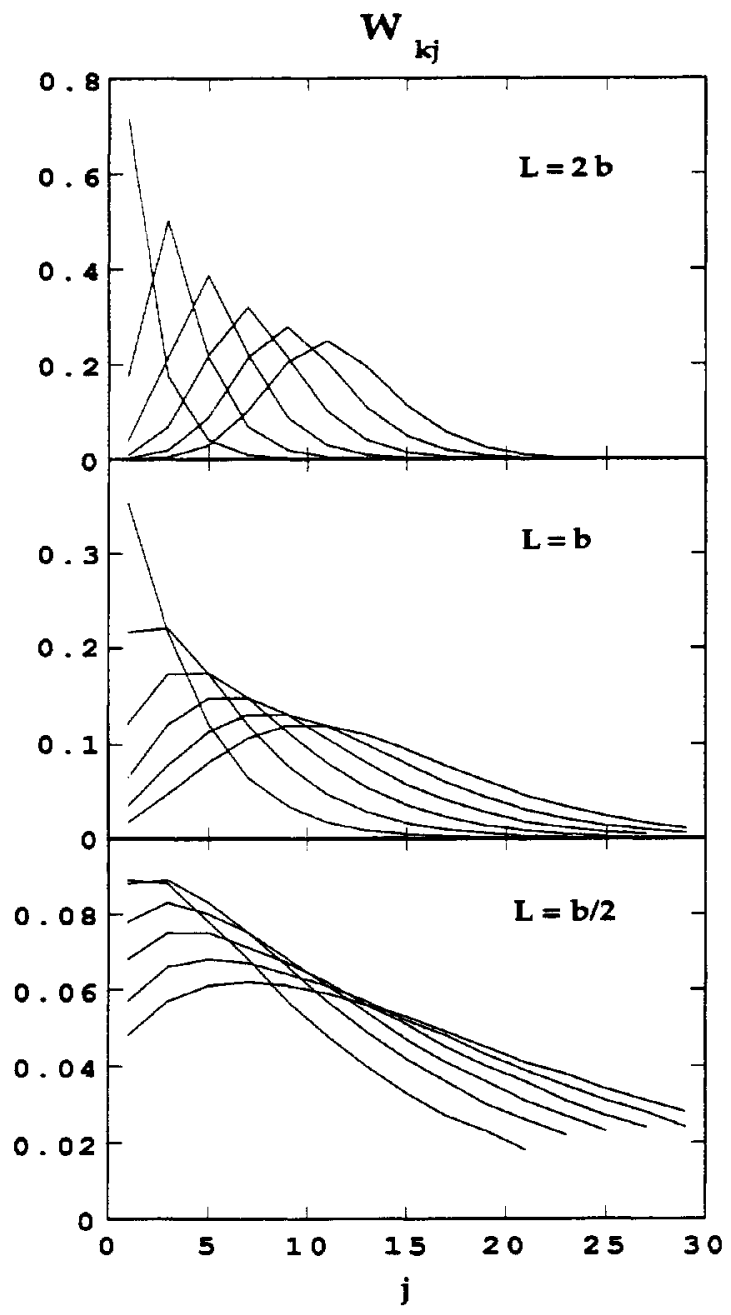

Fig. 1. Variation of the quantities $W_{k j}$ (eq. (4.3)) with the index $j$, for three values of $L / b$. In each case, the curves correspond to $k=1,3,5,7,9$ and 11 , successively, from the inner most one to the outer most one. The quantities $W_{k j}$ are displayed for odd values of $j$ only (see text).

Performing the calculation of the double commutator and using

$[p, W(r)]=\frac{\hbar}{\mathrm{i}}(\nabla W)$

one readily obtains relation (3.8). One can in this way generate other relations similar to (3.7a) and (3.8a) with any positive power of $\left(E_{j}-E_{k}\right)$. Let us mention that the "energy weighted sum rules" are obtained here for a real local potential, $V(r)$. They can be generalized for non local $V$ and non local $W$ but then they imply more complicated forms of the r.h.s. of relation (3.8a) and similar ones.

In the case of a bounded local function $W(r)$ (which occurs in many physical cases), relation (3.6) sets bound on the values of the diagonal matrix elements, whereas relations (3.3), (3.7) and (3.8) set bounds on the off-diagonal matrix elements and on the variations of the quantities $W_{k j}$ with their indices $k$ and $j$. This means that the diagonal elements of $W_{k j}$ are all bounded and that the off-diagonal elements are constrained by eqs. (3.3), (3.4), and should decrease, when one goes farther and farther off the diagonal, sufficiently fast for the summations (3.7) and (3.8) (or their generalizations) to converge.

Of course, all the conditions (3.2)-(3.4) for non local imaginary parts and (3.2)-(3.8) for local imaginary parts are not sufficient to determine the matrix $W_{k j}$, but they have to be fulfilled if one wants the matrix $W_{k j}$ to correspond to a positive definite operator.

\section{Illustrative cases}

To give an idea of the typical variation of $W_{k j}$ with its indices, we have calculated the matrix elements in two simple but illustrative cases. The first one corresponds to $V$ being an infinite square well in one dimension $\left(-R_{0} \leqslant x \leqslant\right.$ $\left.R_{0}\right)$ and $W$ being a step function, i.e. $W(x)=W_{0} \theta(L-|x|)$. One then obtains $\left(L<R_{0}\right)$

$W_{k j}=\frac{W_{0}}{\pi}\left[\frac{\sin \left[\frac{\pi L}{R_{0}}(k-j)\right]}{k-j}+\frac{\sin \left[\frac{\pi L}{R_{0}}(k+j+1)\right]}{k+j+1}\right]$

for the positive parity states $(k, j=0,1,2, \ldots)$, and

$W_{k j}=\frac{W_{0}}{\pi}\left[\frac{\sin \left[\frac{\pi L}{R_{0}}(k-j)\right]}{k-j}-\frac{\sin \left[\frac{\pi L}{R_{0}}(k+j)\right]}{k+j}\right]$

for the negative parity states $(k, j=1,2,3, \ldots)$. If $k=j$, the first term in the parenthesis of both eq. (4.1) and (4.2) should be replaced by $\pi L / R_{0}$. Relation (4.2) also applies to the three-dimensional case for $l=0$ waves.

The second case corresponds to the harmonic oscillator in one dimension, $V=\frac{1}{2} m \omega^{2} x^{2}$ and $W(x)=W_{0} \mathrm{e}^{-x^{2} / L^{2}}$. One then obtains

$$
\begin{aligned}
W_{k j}= & W_{0} \frac{\Gamma\left(\frac{k+j+1}{2}\right)}{\sqrt{2 \pi k ! j !}} \frac{\left(1-2 \alpha^{2}\right)^{(k+j) / 2}}{\alpha^{k+j+1}} \\
& \times{ }_{2} F_{1}\left(-k,-j ; \frac{1-k-j}{2} ; \frac{\alpha^{2}}{2 \alpha^{2}-1}\right),
\end{aligned}
$$

where ${ }_{2} F_{1}$ is the hypergeometric function and where $\alpha^{2}=$ $\frac{1}{2}\left(1+b^{2} / L^{2}\right), b$ being the oscillator characteristic length: $b=\sqrt{(h / m \omega)}$. Formula (4.3) applies when $k+j$ is even, otherwise $W_{k j}$ vanishes.

For the square well, the quantities $W_{k j}$ decrease slowly, when departing from the diagonal $(k=j)$, i.e. when $k$ is fixed and $j(>k)$ increases, oscillating between a maximum, behaving as $1 / j$ as $j$ increases, and a minimum of opposite sign. It is remarkable that this behaviour is barely sufficient to ensure the convergence of the summation in (3.7a). For the harmonic oscillator, when $k$ is fixed and $j$ is increasing, $W_{k j}$ decreases as

$W_{k j} \sim\left(\frac{2 \alpha^{2}-1}{2 \alpha^{2}}\right)^{j / 2}$

the quantity in parenthesis always being smaller than unity. The quantities (4.3) are displayed in Fig. 1. These examples illustrate the fact that the elements of the matrix $W_{k j}$ are decreasing when going further and further apart from the diagonal, as eq. (3.7a) implies. They also suggest that the large $j$ behaviour (for fixed $k$ ) depends upon the ranges of the real and imaginary potentials.

\section{Weak and strong imaginary couplings}

\subsection{Introduction}

In many cases of physical interest [4-6], one usually calculates the eigenfunctions $\psi_{j}$ by solving the Schrödinger equation for the real part of the Hamiltonian $H_{0}$ (see eq. (2.2)) in 
$r$-space and the widths of the bound states are very often calculated by using the popular weak coupling formula

$\frac{\Gamma_{k}}{2}=\left\langle\psi_{k}|W| \psi_{k}\right\rangle=W_{k k}$.

It is very hard to discuss the validity of this formula in all generality. It is better to look at this problem when it is formulated in state representation (eq. (2.3) and (2.4)), since then the weak coupling approximation amounts to neglecting the off-diagonal matrix elements of the matrix $W_{k j}$. Of course, some difficulties appear with this representation. One is inevitably led to truncate the (in principle) infinite matrix $W_{k j}$, for practical reasons. The size of the matrix which would correspond to the same accuracy as the standard techniques of solving Schrödinger equation in $r$-space, is presumably very large. Therefore, it is certainly hopeless to investigate the effect of the off-diagonal elements in all generality. In our opinion, it is, however, worthwhile to study the two level case first. This may appear to be only academic, since it would be very exceptional if the dynamics would decouple two of the $\psi_{j}$ states from the rest. Nevertheless, we believe that some features of the two level case, that we outline below, may persist in some form in the general problem, and that may help us to study the validity of the weak coupling, both in the static and the time-dependent cases.

\subsection{The static two level case}

The eigenvalue problem (2.5) takes then the simple form $\left(E_{1}>E_{2}\right)$

$\left(\begin{array}{cc}E_{1}-E-\mathrm{i} W_{11} & -\mathrm{i} W_{12} \\ -\mathrm{i} W_{12} & E_{2}-E-\mathrm{i} W_{22}\end{array}\right)\left(\begin{array}{l}c_{1} \\ c_{2}\end{array}\right)=0$

As we explained in section 3 , if we want the model defined in eq. (2.5) to correspond to a negative definite imaginary absorption, the quantities $\mathrm{W}_{k j}$ are bound to fulfill some constraints. In the two level case (eq. (5.2)), these constraints reduce to the non negativity of $W_{11}$ and Schwarz's inequality

$W_{12}^{2} \leqslant W_{11} W_{22}$,

which requires the non negativity of $W_{22}$. It is convenient to express the quantities in terms of reduced parameters

$\varepsilon=\frac{E_{1}+E_{2}}{E_{1}-E_{2}}, \quad x_{+}=\frac{W_{11}+W_{22}}{E_{1}-E_{2}}, \quad x_{-}=\frac{W_{11}-W_{22}}{E_{1}-E_{2}}$,

$y=\frac{2 W_{12}}{E_{1}-E_{2}}$.

The parameter

$\rho=x_{+}-\sqrt{x_{-}^{2}+y^{2}}$

expresses the conformity to Schwarz's inequality. Indeed, it can be rewritten as

$$
\begin{aligned}
\rho= & \frac{1}{E_{1}-E_{2}}\left[\left(W_{11}+W_{22}\right)\right. \\
& \left.-\sqrt{\left(W_{11}+W_{22}\right)^{2}+4\left(W_{12}^{2}-W_{11} W_{22}\right)}\right] .
\end{aligned}
$$

When Schwarz's inequality is violated, $\rho$ is negative. The eigenvalues are given by

$\mathscr{E}_{1,2}=\frac{\left|E_{1}-E_{2}\right|}{2}\left\{\varepsilon-\mathrm{i}\left(\sqrt{x_{-}^{2}+y^{2}}+\rho\right) \pm \sqrt{\left(1-\mathrm{i} x_{-}\right)^{2}-y^{2}}\right\}$

The advantage of expression (5.6) is the fact that criterion (5.3) enters through the $\rho$ parameter only: hence, $x_{-}, y$ and $\rho$ can be varied independently, provided one restricts to positive values of $\rho$.

Let us first concentrate on the widths $\Gamma_{k}$, i.e. minus twice the imaginary parts of the eigenvalues

$\frac{\Gamma_{1,2}}{\left|E_{1}-E_{2}\right|}=\left\{\left(\sqrt{x_{-}^{2}+y^{2}}+\rho\right) \pm \operatorname{Im} \sqrt{\left(1-\mathrm{i} x_{-}\right)^{2}-y^{2}}\right\}$.

The typical variations of the widths with respect to the parameters $y$ and $x_{-}$(corresponding to the difference of $W_{11}$ and $W_{22}$ ) are given in Fig. 2 , for $\rho=0$, i.e. for the maximum non diagonal coupling just fulfilling requirement (5.3). The remarkable result is the forbidden region indicated by the shaded area, It is easy to trace back the mathematical origin of this forbidden region. Let us consider $x_{-}=0$, for simplicity. The square root in (5.7) has no imaginary part as far as $y^{2} \leqslant 1$ : in that particular case, the two widths are identical and are represented by the diagonal in Fig. 2. As soon as $y$ is larger than unity, the two eigenvalues start to differ. Figure 2 tells that, whatever the value of $x_{-}$, when the coupling $(y)$ becomes too strong, one of the imaginary parts grows at the expanse of a decrease of the other. The forbidden region is thus an intrinsic property of the complex coupling.

When one allows for a departure from $\rho=0$, but in concordance with Schwarz's inequality, i.e. $\rho>0$, the pattern does not change. There is a mere shift of the curves towards the bottom of Fig. 2, i.e. a uniform increase of the imaginary parts. When Schwarz's inequality is violated, $\rho<0$, the curves in Fig. 2 are shifted to the top and at least one of the

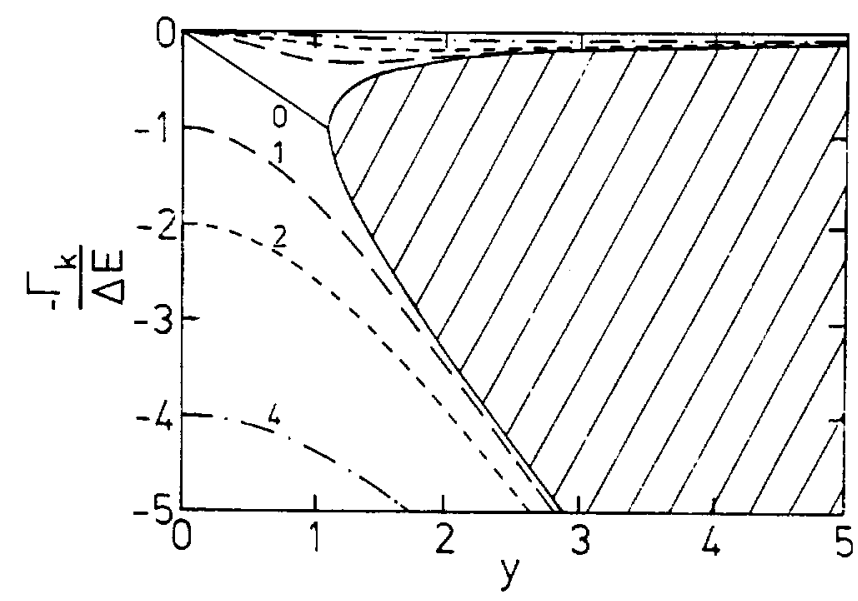

Fig. 2. Level widths (divided by $\Delta E=E_{1}-E_{2}$ ) for the two level case and for $\rho=0$ (the maximum coupling compatible with Schwarz's inequality), as function of the parameters $y$ and $x_{-}$, whose values are indicated close to the corresponding curves. Note that for $x_{-} \neq 0$, there are two curves, represented by the same symbol. For $x_{-}=0$ (full curves) the two curves coalesce up to $y=1$ and diverge for higher values. The shaded area indicates the forbidden values of the widths. 
imaginary parts can become positive. Therefore, in the two level case, Schwarz's inequality is equivalent to a sufficient criterion for having absorption only and not creation of flux, whatever the values of the parameters, as we explained in Section 3.

The role of Schwarz's inequality (or more generally of conditions $(3.2)-(3.4), \ldots)$ is thus rather clear. Would one wish to study the coupling of bound states in $H_{0}$ with other channels by model (2.5) from the very beginning, arbitrary values of the matrix $W_{k j}$ are not permitted. In the two level case, only those consistent with Schwarz's inequality (and of course with the positive definiteness of $W_{11}$ and $W_{22}$ ) would lead to the same physics as a negative definite imaginary potential, i.e. to pure absorption.

The introduction of the parameter $\rho$ is very convenient to exhibit the consequences of Schwarz's inequality, but for the subsequent discussion, it is preferable to stick with the parameters $x_{+}, x_{-}$and $y$. The widths are then given by

$\frac{\Gamma_{1,2}}{\left(E_{1}-E_{2}\right)}=x_{+} \pm \operatorname{Im} \sqrt{\left(1-\mathrm{i} x_{-}\right)^{2}-y^{2}}$.

They are represented in Fig. 3, which actually displays the 1.h.s. of eq. (5.8) after subtraction of $x_{+}$, i.e. the average of $W_{11}$ and $W_{22}$, divided by $E_{1}-E_{2}$ (see eq. (5.4)). The forbidden zone in this representation is also visible. The limitations imposed by Schwarz's inequality take the form $y \leqslant$ $\sqrt{x_{+}^{2}-x_{-}^{2}}$, i.e. restrict the allowed values of $y$, for fixed $x_{+}$ and $x_{-}$. It can be checked that, under these conditions, the quantities $\Gamma_{i}$ are positive.

Let us say a few words on the real parts of the complex eigenvalues. They are given by

$\operatorname{Re} \mathscr{E}_{1,2}=\frac{E_{1}-E_{2}}{2}\left\{\varepsilon \pm \operatorname{Re} \sqrt{\left(1-\mathrm{i} x_{-}\right)^{2}-y^{2}}\right\}$

or

$\frac{\operatorname{Re} \mathscr{E}_{1,2}-\frac{E_{1}+E_{2}}{2}}{E_{1}-E_{2}}= \pm \frac{1}{2} \operatorname{Re} \sqrt{\left(1-\mathrm{i} x_{-}\right)^{2}-y^{2}}$.

They are thus symmetric with respect to $\left(E_{1}+E_{2}\right) / 2$. The numerical values are given in Fig. 4. The most remarkable result is that the imaginary coupling introduces an attrac-

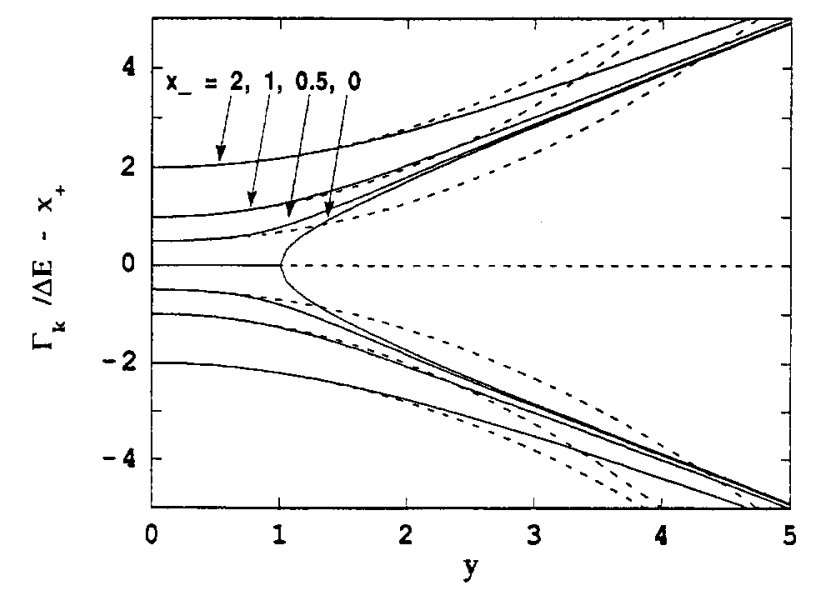

Fig. 3. Level widths (divided by $\Delta E=E_{1}-E_{2}$ prior subtraction of $x_{+}$), as functions of the parameters $y$ and $x_{-}$, whose values are indicated. The exact values of the widths (eq. (5.8)) are given by the full curves, while the dotted curves correspond to approximation (5.11).

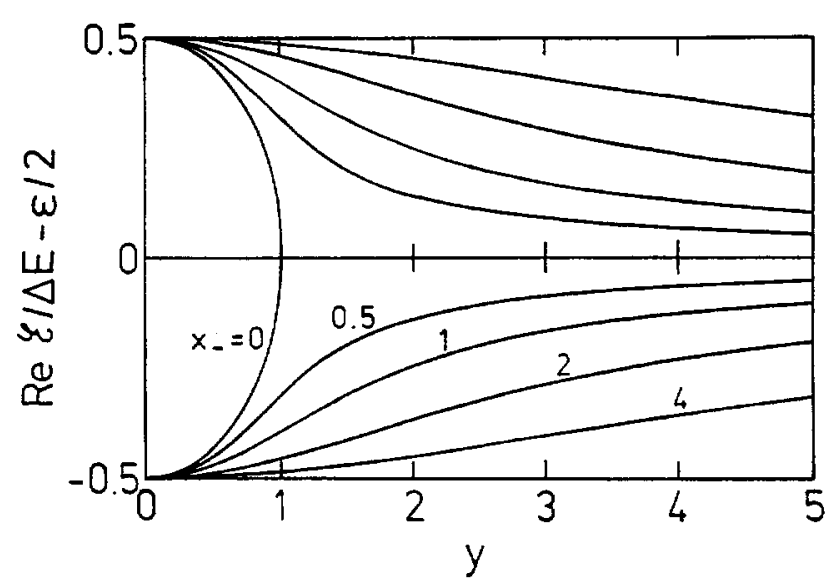

Fig. 4. Real part of the eigenvalues for the two level case (with respect to the mean value of the unperturbed energies, see eq. (5.9b)), as functions of the parameters $y$ and $x_{-}$, whose value is indicated close to the corresponding curve. For $x_{-}=0$ and $y>1$, the two real parts are vanishing.

tion of the eigenvalues, in contrast with the real coupling case which produces a repulsion of the eigenvalues [15]. For $x_{-}=0$, i.e. for $W_{11}=W_{22}$, the two eigenvalues coalesce for $y>1$ (the quantity (5.9b) then vanishes). For $x_{-} \neq 0$, they almost coalesce for large values of $y$.

In summary, we found the following non trivial behaviour of the eigenvalues: (i) the real parts are pulled toward one another by the imaginary coupling (level attraction); (ii) these are forbidden regions for the imaginary parts of the eigenvalues, whose consequence is the growth of one of the imaginary parts at the expanse of the other when the imaginary coupling is increased. It is more appropriate to say that there are strictly forbidden values of the difference of the widths at strong coupling, as illustrated by Figs 2 and 3 .

We conjecture that these results hold qualitatively in the general (many level) case. Indeed, property (i) holds for an imaginary coupling of any strength. It is opposite to the result for real coupling case and it is well known that level repulsion occurs in the many-level case, giving rise to a larger dispersion of the eigenstates. We emphasize that the level attraction linked to the imaginary coupling should be viewed as a mathematical property. In an actual physical case, when the underlying full many-channel problem is solved with a real coupling, only level repulsion occurs. However, when the full problem is reduced to the equivalent one-channel problem with a complex coupling, the real part of the coupling would lead to a too strong level repulsion, which would be corrected by the level attraction due to the imaginary coupling. Property (ii) is a genuine property of the imaginary coupling. If this property holds for the manylevel case, it may lead in the strong coupling case to a strong increase of one or a few widths. We have verified (see below) on a specific case that the properties of the decay of an eigenstate of the real part of the Hamiltonian (mode mixing) are still valid on a more general case. The correlated decrease of the remaining widths should be reflected by an enhancement of the small width part of the width distribution in a small energy interval. This is embodied by the socalled Porter-Thomas distribution for the widths [16], and verified experimentally [17]. As far as we know, the possible link between the results of the above two state model and the Porter-Thomas distribution has never been discussed or alluded to. 


\subsection{Weak coupling}

As we said above, we are interested in knowing under which conditions the widths can be accurately estimated by the first order (in the diagonal coupling) formula (5.1). It is very hard to answer this question in all generality with arguments based on perturbation theory. Indeed, the next order approximation to $\Gamma_{k}$ in terms of the non diagonal matrix elements $W_{k j}$ (i.e. including the diagonal elements in the unperturbed energies), is given by

$\frac{\Gamma_{k}}{2}=W_{k k}-\sum_{j \neq k} \frac{W_{k j}^{2}\left(W_{k k}-W_{j j}\right)}{\left(E_{k}-E_{j}\right)^{2}+\left(W_{k k}-W_{j j}\right)^{2}}$,

and therefore implies complicated properties of the $W_{k j}$ 's and of the unperturbed spectrum. The two level case can thus be of some help to illustrate the situation when a level is perturbed by its very near neighbors only.

In the two level case, approximation (5.11) corresponds to the first order approximation in $y^{2}$ in eq. (5.8), keeping $x_{+}$ and $x_{\text {- }}$ fixed, while approximation (5.1) corresponds to $y=0$. Approximation (5.11) is compared to the exact result in Fig. 3. It is quite accurate up to $y=2 W_{12} /\left(E_{1}-E_{2}\right) \lesssim 0.5$, irrespective of the value of $x_{-}$and surprisingly good for larger values of the coupling $y$, when $x_{-}$is large. Note however the complete failure for $x_{-}=0$ and $y>1$, where the problem becomes suddenly non perturbative.

The second term in (5.11) gives also an idea of the accuracy of the weak coupling approximation (5.1). In the two level case, it is equal to $\left.x_{-} y^{2} / 1+\left(x_{-}\right)^{2}\right)$. It is interesting to note that for a given value of $y^{2}$, this term is small when $x_{-}$ is either very small or very large, and is the largest for $x_{-}=$ 1.

From the last result, it may be expected that the first correction to (5.1), i.e. the second term in eq. (5.11), may be considered as providing an accurate result, as far as the equivalent of the $y$ parameter for the many level case is $\lesssim$ 0.5 . We propose the following straightforward extension of the $y$ parameter

$y=2 \sum_{j \neq k} \frac{\left|W_{k j}\right|}{E_{k}-E_{j}}$

In summary, one expects that when the last quantity is $\lesssim$ 0.5 , expression (5.11) is quite accurate.

\subsection{Time-dependent problem}

We want here to investigate the time evolution of the wave function $\psi(r, t)$ (eq. (2.1)) when one starts at $t=0$ with an eigenstate of $H_{0}$ (eq. (2.2)), or equivalently the time evolution of the coefficients $c_{k}(t)$ (eq. (2.3)) when, at $t=0$, all of them vanish but one, which is equal to unity.

Let us first start with the two level case, with $c_{1}$ $(t=0)=1$ and $c_{2}(t=0)=0$. As we explained in section 5.2 , the two relevant parameters are $x_{-}$and $y$. So we illustrate the typical behaviours of $\left|c_{1}\right|^{2}$ and $\left|c_{2}\right|^{2}$ for various values of the parameters $x_{-}$and $y$ in Fig. 5. They can be understood when one notices that the general solution of eq. (2.3) can be written as $(h=1)$

$\left(\begin{array}{l}c_{1}(t) \\ c_{2}(t)\end{array}\right)=\alpha_{1}\left(\begin{array}{l}d_{1} \\ d_{2}\end{array}\right) \mathrm{e}^{-\mathrm{i} \tilde{\delta}_{1} t}+\alpha_{2}\left(\begin{array}{l}b_{1} \\ b_{2}\end{array}\right) \mathrm{e}^{-\mathrm{i} \mathscr{E}_{2} t}$,

where $\varepsilon_{1,2}$ are the complex eigenvalues (5.6), $\left(\begin{array}{l}d_{1} \\ d_{2}\end{array}\right)$ and $\left(\begin{array}{l}b_{1} \\ b_{2}\end{array}\right)$ are the corresponding eigenvectors and where $\alpha_{1}$ and $\alpha_{2}$ are
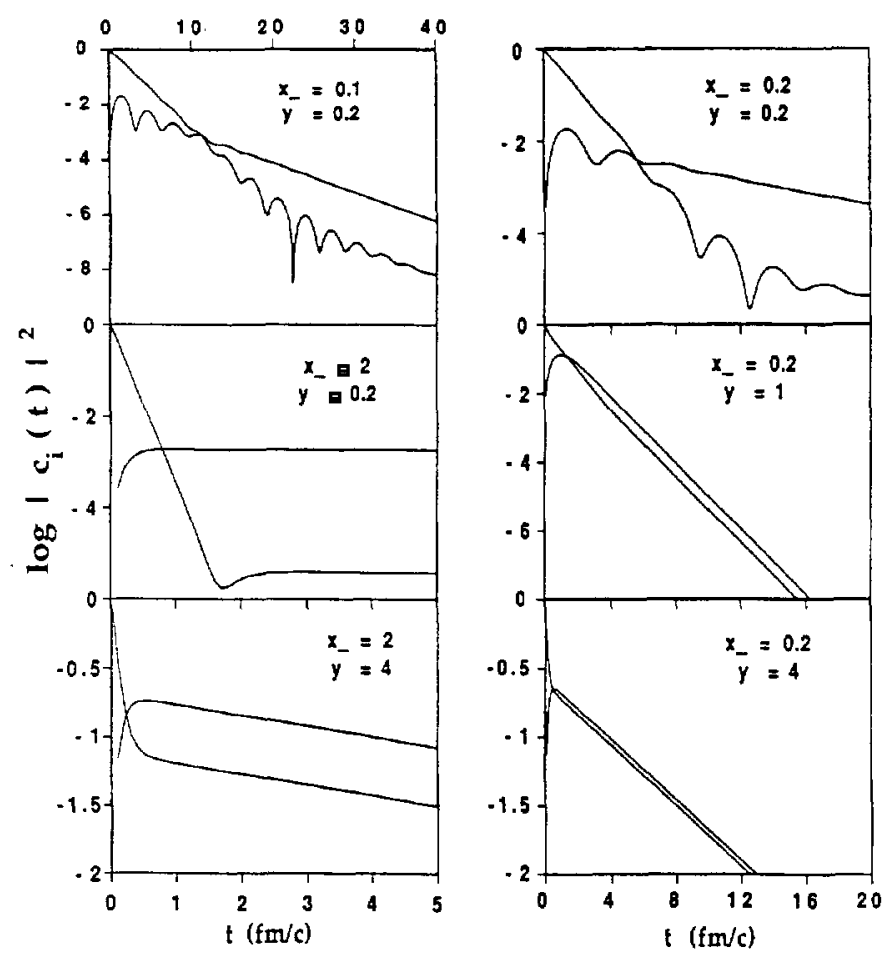

Fig. 5. Time evolution of the $\left|c_{i}(t)\right|^{2}$ (eq. (5.13)) for various values of the parameters $x_{-}$and $y$ (and $\rho=0$ ) in the two level case. Note the different vertical scales and the different time scale for the upper left part of the figure, indicated at the top of the figure.

standing to guarantee the initial conditions. Therefore, one has

$$
\begin{aligned}
\left|c_{1}(t)\right|^{2} & =\left|\alpha_{1}\right|^{2}\left|d_{1}\right|^{2} \mathrm{e}^{-\Gamma_{1} t}+\left|\alpha_{2}\right|^{2}\left|b_{1}\right|^{2} \mathrm{e}^{-\Gamma_{2} t} \\
& +2 \operatorname{Re}\left(\alpha^{*}{ }_{1} d^{*}{ }_{1} \alpha_{2} b_{1} \mathrm{e}^{\mathrm{i} \operatorname{Re}\left(\mathscr{E}_{1}-\varepsilon_{2}\right) t} \mathrm{e}^{\left.-\left(\Gamma_{1}+\Gamma_{2}\right) t / 2\right) .}\right.
\end{aligned}
$$

Note that one has $\alpha_{1} d_{1}+\alpha_{2} b_{1}=1$. The variation of $\left|c_{1}(t)\right|^{2}$ shows the two exponential decays due to the first two terms in eq. (5.14), plus oscillations (of quantum nature) due to the last term in eq. (5.14) which are damped rapidly if the widths are sufficiently large. The oscillations are the most important when $y \leqslant 1$ and $x_{-}$is close to zero. One can see that in all cases, the quantity $\left|c_{1}(t)\right|^{2}$ decreases exponentially by several orders of magnitude before changing of slope, except for very strong coupling. Therefore, the strong coupling does not only change the imaginary parts of the eigenvalues (compared to simplest approximation (5.1)), but also introduces a rapid turn-over from one decay mode to the other one. Let us notice that in the weak coupling approximation (5.1), $\left|c_{1}(t)\right|^{2}$ is purely exponential and $c_{2}(t)$ vanishes.

In Fig. 6, we illustrate the behaviour of the $\left|c_{i}(t)\right|^{2}$ for a more realistic problem: the charmonium model of Ref. [7]. The potential $V(r)$ is the usual charmonium potential and $W(r)$ is taken as a constant value, $W_{0}$, for $r>1.0 \mathrm{fm}$ and zero below, reflecting the idea that a quark-antiquark string breaks into a $D-\bar{D}$ system, if the length is long enough. At $t=0$, the system is supposed to be in the so-called $\psi^{(2)}$ state, and then evolves under the imaginary coupling. Equation (2.1) is solved with a very large mesh in $r$-space (step size $\approx 0.01 \mathrm{fm}$ ), which amounts to solve (after appropriate projection yielding the coefficients $\left.c_{i}(t)\right)$ eq. (2.3) in a very large basis. When the coupling is small, the initial state decays exponentially several orders of magnitude before a change of slope takes place. When the coupling is strong, the various modes are mixed more rapidly. It is remarkable 


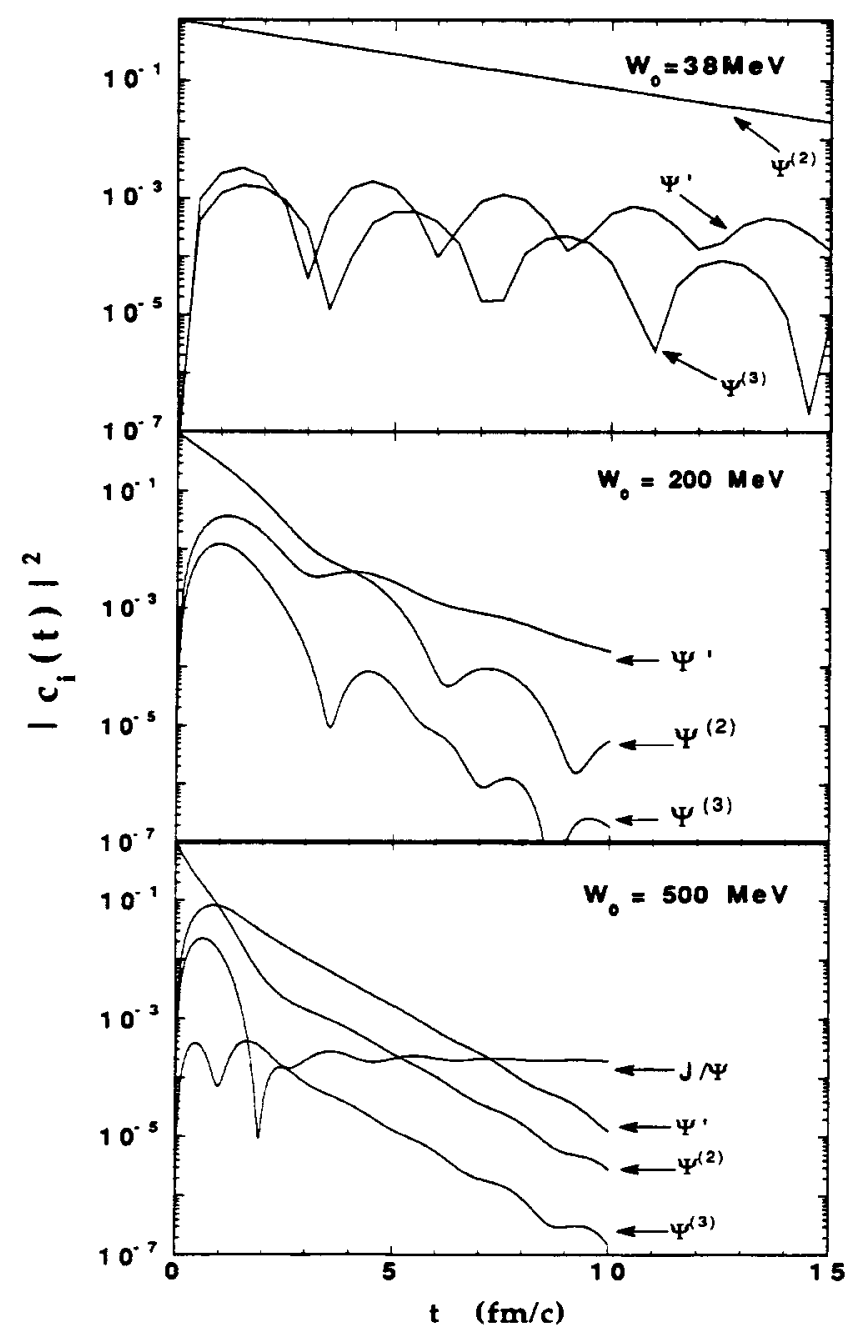

Fig. 6. Time evolution of the quantities $\left|c_{i}(t)\right|^{2}$ for the case of the charmonium system (see text and Ref. [7]), for various values of the imaginary coupling parameter $W_{0}$.

that the typical behaviours observed in the two level case are reproduced in this realistic case. If one considers the two states $\psi^{(2)}$ and $\psi^{\prime}$ only, the cases illustrated by the central and bottom part of Fig. 6 correspond to $x_{-}=0.23$ and $y=0.39$ and to $x_{-}=0.58$ and $y=0.98$, respectively. There is indeed a strong resemblance, as far as the variation of the quantity $\left|c_{i}(t)\right|^{2}$ is concerned, with respectively the upper and central parts of Fig. 5 (second column), for which the values of the coefficients $x_{-}$and $y$ are similar. Let us finally mention that the first value of the imaginary strength, $W_{0}$, is the closest to the actual physical value [7].

\section{Properties of $W\left(r, r^{\prime}\right)$ associated with a diagonal imaginary coupling matrix}

In this section, we make some remarks on the (reverse) problem stated in the end of section 2 . Let the effective bound state problem be expressed by eq. (2.6) in the representation of the eigenstates of the real one-body Hamiltonian. We first here list the main properties of the corresponding imaginary potential (see also eqs. (2.7) and $(2.8))$,

$W\left(\boldsymbol{r}, \boldsymbol{r}^{\prime}\right)=\sum \frac{\Gamma_{j}}{2} \psi_{j}(\boldsymbol{r}) \psi_{j}\left(\boldsymbol{r}^{\prime}\right)$

to be added to the real part of the one-body Hamiltonian, in order to reproduce a diagonal imaginary coupling matrix. They are:

(1) $W\left(r, \boldsymbol{r}^{\prime}\right)=W\left(\boldsymbol{r}^{\prime}, \boldsymbol{r}\right), \quad W(\boldsymbol{r}, \boldsymbol{r}) \geqslant 0$.

(2) $\int W(\boldsymbol{r}, \boldsymbol{r}) \mathrm{d}^{3} r=\frac{1}{2} \sum_{j} \Gamma_{j}$.

This results from the invariance of the trace of an operator.

(3) $\int\left[W\left(r, r^{\prime}\right)\right]^{2} \mathrm{~d}^{3} r^{\prime}=\frac{1}{4} \sum_{j} \Gamma_{j}^{2} \psi_{j}^{2}(r)$.

(4) $\iint\left[W\left(r, r^{\prime}\right)\right]^{2} \mathrm{~d}^{2} r \mathrm{~d}^{3} r^{\prime}=\frac{1}{4} \sum_{j} \Gamma_{j}^{2}$.

The latter proceeds from the invariance of the norm.

(5) $\left[W\left(\boldsymbol{r}, \boldsymbol{r}^{\prime}\right)\right]^{2} \leqslant W(\boldsymbol{r}, \boldsymbol{r}) W\left(\boldsymbol{r}^{\prime}, \boldsymbol{r}^{\prime}\right)$.

This is a consequence of Cauchy's inequality [10].

These conditions are resting on the positivity of the $\Gamma_{j}$ 's. Other properties are related with the variations of the $\Gamma_{j}$ 's with the indices $j$. Indeed, if all the $\Gamma_{j}$ 's are equal, $W\left(r, r^{\prime}\right)$ is diagonal. Of course, this also depends upon the eigenfunctions $\psi_{j}$. So a general discussion is out of scope. We illustrate the non locality of $W\left(r, r^{\prime}\right)$ in the case of a threedimensional infinite square well of radius $R_{0}$ for $l=0$ waves. The corresponding multipole of $W\left(\boldsymbol{r}, \boldsymbol{r}^{\prime}\right)$ writes

$W\left(r, r^{\prime}\right)=\frac{2}{R_{0}} \frac{1}{4 \pi} \sum_{n=1}^{\infty} \frac{\Gamma_{n}}{r r^{\prime}} \sin \frac{n \pi r}{R_{0}} \sin \frac{n \pi r^{\prime}}{R_{0}}$.

Introducing the coordinates $R=\left(r+r^{\prime}\right) / 2, s=r-r^{\prime}$, (with $0 \leqslant R \leqslant R_{0}, 0 \leqslant s \leqslant 2 R_{0}$ ), one obtains

$$
\begin{aligned}
W\left(r, r^{\prime}\right)= & -\frac{1}{4 \pi R_{0}} \frac{1}{R^{2}-\frac{s^{2}}{4}} \\
& \times \sum_{n=1}^{\infty} \Gamma_{n}\left[\cos \left(\frac{n \pi 2 R}{R_{0}}\right)-\cos \left(\frac{n \pi s}{R_{0}}\right)\right] .
\end{aligned}
$$

Let us consider the three following cases:

(1) $\Gamma_{n}=\Gamma / n$. One then has:

$W\left(r, r^{\prime}\right)=\frac{\Gamma}{8 \pi R_{0}\left(R_{0}^{2}-\frac{s^{2}}{4}\right)} \ln \left|\frac{1-\cos \frac{\pi s}{R_{0}}}{1-\cos \frac{2 \pi R}{R_{0}}}\right|$.

In this case, $W\left(r, r^{\prime}\right)$ is infinite for $r=r^{\prime}$, but rapidly decreases as $s$ increases, like

$W\left(r, r^{\prime}\right) \sim \ln \left(\frac{s}{R_{0}}\right)$.

(2) $\Gamma_{n}=\Gamma / n^{2}$. One can then write

$W\left(r, r^{\prime}\right)=\frac{1}{4 \pi R_{0}} \frac{\Gamma}{R^{2}-\frac{s^{2}}{4}}\left[\frac{\pi^{2} R}{R_{0}}-\frac{\pi^{2} R^{2}}{R_{0}^{2}}-\frac{\pi^{2}|s|}{2 R_{0}}+\frac{\pi^{2} s^{2}}{4 R_{0}^{2}}\right]$.

In this case, $W\left(r, r^{\prime}\right)$ is finite for $r=r^{\prime}$ as it should when $\sum_{j} \Gamma_{j}$ converges (see eq. (6.2)), and for small $s$, the behaviour is the following

$W\left(r, r^{\prime}\right) \sim 1-\frac{R_{0}}{R\left(R-R_{0}\right)}|s|$. 
(3) $\Gamma_{n}=\Gamma / n^{4}$. One has, in this case,

$$
\begin{aligned}
W\left(r, r^{\prime}\right) & =\frac{1}{4 \pi R_{0}} \frac{\Gamma}{R^{2}-\frac{s^{2}}{4}} \\
\times & {\left[\frac{\pi^{4} R^{2}}{3 R_{0}^{2}}\left(1-\frac{R}{R_{0}}\right)^{2}-\frac{\pi^{4} s^{2}}{12 R_{0}^{2}}\left(1-\frac{|s|}{2 R_{0}}\right)^{2}\right] . }
\end{aligned}
$$

The behaviour for small $s$ is given by

$W\left(r, r^{\prime}\right) \sim 1-\frac{s^{2} R_{0}^{2}}{4 R^{2}\left(R_{0}-R\right)^{2}}$.

One sees that the form of the non locality (i.e. the formal analytic dependence upon $s$ for small $s$ ) is changing with the exponent of $n$ in the three cases mentioned above. It can be proved that the quadratic dependence on survives for all powers larger or equal to 4 . This formal dependence is also the one which is phenomenologically considered (see Ref. [1]), as a gaussian form in $s$ is often used.

It is also interesting to consider the case $\Gamma_{n}=\Gamma a^{n}$, with $a<1$. One then obtains

$$
\begin{aligned}
W\left(r, r^{\prime}\right) & =-\frac{1}{4 \pi R_{0}} \frac{\Gamma}{R^{2}-\frac{s^{2}}{4}} \\
\times & {\left[\frac{1-a \cos \left(\frac{2 \pi R}{R_{0}}\right)}{1-2 a \cos \left(\frac{2 \pi R}{R_{0}}\right)+a^{2}}-\frac{1-a \cos \left(\frac{\pi s}{R_{0}}\right)}{1-2 a \cos \left(\frac{\pi s}{R_{0}}\right)+a^{2}}\right] }
\end{aligned}
$$

For small s, one obtains the following behaviour

$$
\begin{aligned}
W\left(r, r^{\prime}\right) & \propto 1-\frac{s^{2}}{4 R^{2}} \\
& \times\left\{\left(\frac{2 \pi R}{R_{0}}\right)^{2} \frac{\left(1-2 a \cos \left(\frac{2 \pi R}{R_{0}}\right)+a^{2}\right)}{2(1-a)^{2}\left(1-\cos \left(\frac{2 \pi R}{R_{0}}\right)\right)}-1\right\} .
\end{aligned}
$$

The usual quadratic dependence with a negative coefficient is also obtained. Furthermore, the last expression clearly indicates that the faster $\Gamma_{n}$ decreases when $n \rightarrow \infty$ (i.e. for smaller and smaller values of $a$ ), the larger is the non locality.

\section{Conclusion}

We have studied the complex coupling between bound states in an effective one particle Hamiltonian. This situation is often encountered in physics, when a many channel problem cannot be solved and when the approach is restricted to a one channel problem with an effective interaction. We studied this problem when the coupling occurs through a negative definite imaginary potential. We have exhibited the properties of the corresponding imaginary matrix when the problem is formulated in the representation of the eigenstates of the real part of the Hamiltonian.

We have studied the simple but illuminating two level case and underlined the importance of Schwarz's inequality which constrains the value of the non diagonal coupling in the state representation. Non trivial results, as level attraction and excluded values for the difference of the widths have been worked out.

We have also delineated for the two level case the accuracy of the weak coupling approximation (5.1) to the widths (in the first order of the diagonal coupling, i.e. in zeroth order in the off-diagonal coupling). It is surprisingly good in a large domain of the $\left(x_{-}, y\right)$ parameter plane, especially for largely different diagonal elements (large $x_{-}$). Furthermore, the first correction to (5.1), i.e. the second term in (5.11), gives a good approximation to the exact result in a wider range of the $\left(x_{-}, y\right)$ plane. We proposed a qualitative criterion for a general situation.

We also investigated the time evolution of an eigenstate of the real Hamiltonian in the presence of the imaginary coupling. We have shown that decay mode mixing and quantum interferences are consequences of the imaginary coupling, and disappear in the limit of weak coupling. We displayed in one physical case, the interplay of several decay modes.

Finally, we investigated the properties of the imaginary potential in $\boldsymbol{r}$-space, corresponding to a diagonal imaginary matrix in the state representation. We derived a qualitative relation between the variation of the elements of the matrix and the non locality of the corresponding imaginary potential.

\section{Acknowledgements}

This work was supported by contract SPPS-IT/SC/29 and by the FNRS, Belgium.

\section{References}

1. Hodgson, P. E., "Nuclear Reactions and Nuclear Structure", (Clarendon Press, Oxford, 1971).

2. Feshbach, H., Ann. Phys. (N.Y.) 5, 357 (1958); 19, 287 (1962).

3. Mahaux, C. and Weidenmüller, H. A., "Shell-model Theory of Nuclear Reactions" (North-Holland, Amsterdam, 1969).

4. Hüfner, J., Phys. Rep. 21, 1 (1975).

5. Batty, C. J., Sov. J. Part. Nucl. 13, 71 (1982).

6. Batty, C. J., Rep. Prog. Phys. 52, 1165 (1989).

7. Cugnon, J. and Gossiaux, P.-B., Europhys. Lett. 20, 31 (1992)

8. Cugnon, J. and Gossiaux, P.-B., Z. Physik C58, 77 (1993).

9. Fano, U., Phys. Rev. 124, 1866 (1961).

10. Schiff, L. J., Quantum Mechanics, 3rd ed., (McGraw-Hill, Tokyo, 1968), ch. 6.

11. Hohn, F. E., "Elementary Matrix Algebra", 3rd ed., (The Macmillan Company, New York, 1973), ch. 10.

12. Abramowitz, M. and Stegun, I. A., "Handbook of Mathematical Functions", (Dover, New York, 1972), p. 11.

13. Lane, A. M., "Nuclear Theory", (Benjamin, New York, 1964).

14. Noble, J. V., Ann. Phys. (N.Y.), 67, 98 (1971); Phys. Rep. C40, 241 (1978)

15. Wigner, E. P., Proc. Conf. on Neutron Physics by Time-of-Flight, Gatlinburg, Oak Ridge National Report ORNL-2309 (1956).

16. Porter, C. and Thomas, R. G., Nucl. Phys. 104, 483 (1956).

17. Lynn, J. E., "The Theory of Neutron Resonances Reactions", (Clarendon Press, Oxford, 1968), ch. 6. 